\title{
Some results about a special nonlinear difference equation and uniqueness of difference polynomial
}

\author{
Jianming $\mathrm{Qi}^{1^{*}}$, Jie Ding ${ }^{2}$ and Taiying Zhu ${ }^{2}$
}

\author{
* Correspondence: \\ qijianmingdaxia@163.com \\ 'Department of Mathematics and \\ Physics, Shanghai Dianji University, \\ Shanghai 200240, PR China \\ Full list of author information is \\ available at the end of the article
}

\begin{abstract}
In this paper, we continue to study a special nonlinear difference equation solutions of finite order entire function. We also continue to investigate the value distribution and uniqueness of difference polynomials of meromorphic functions. Our results which improve the results of Yang and Laine [Proc. Jpn. Acad. Ser. A Math. Sci. 83:50-55 (2007)]; Qi et al. [Comput. Math. Appl. 60:1739-1746 (2010) ].

Mathematics Subject Classification (2000): 30D35, 39B32, 34M05.
\end{abstract}

Keywords: Meromorphic functions, Nevanlinna Theory, difference equation, difference polynomial

\section{Introduction}

Let $f(z)$ be a meromorphic function in the whole complex plane $\mathbb{C}$. It is assumed that the reader is familiar with the standard symbols and fundamental results of Nevanlinna theory such as the characteristic function $T(r, f)$, proximity function $m(r, f)$, counting function $N(r, f)$, the first and second main theorem etc.,(see [1,2]). The notation $S(r, f)$ denotes any quantity that satisfies the condition: $S(r, f)=o(T(r, f))$ as $r \rightarrow \infty$ possibly outside an exceptional set of $r$ of finite linear measure. A meromorphic $a(z)$ is called a small function of $f(z)$ if and only if $T(r, a(z))=S(r, f)$. A polynomial $Q(z, f)$ is called a differential-difference polynomial in $f$ whenever $f$ is a polynomial in $f(z)$, its derivatives and its shifts $f(z+c)$, with small functions of $f$ again as the coefficients. Denote $\Delta_{c}$ $f:=f(z+c)-f(z)$, and $\Delta_{c}^{n} f=\Delta_{c}^{n-1}\left(\Delta_{c} f\right)$ for all $n \in N, n \geq 2$, where $c$ is nonzero complex constant.

Recently, Yang and Laine [3] proved:

Theorem A Let $p$ be a non-vanishing polynomial, and let $b, c$ be nonzero complex numbers. If $p$ is nonconstant, then the differential equation

$$
f^{3}+p(z) f^{\prime \prime}=c \sin b z
$$

admits no transcendental entire solutions, while if $p$ is constant, then the equation admits three distinct transcendental entire solutions, provided $\left(\frac{p b^{2}}{27}\right)^{3}=\frac{1}{4} c^{2}$.

Remark 1. As an example of the case with $p(z)$ constant, recall the nonlinear differential equation

$$
4 f^{3}+3 f^{\prime \prime}=-\sin 3 z
$$


As pointed out by Li and Yang [4], Equation (1.2) admits exactly three distinct transcendental

entire solutions: $f_{1}(z)=\sin z, f_{2}(z)=\frac{\sqrt{3}}{2} \cos z-\frac{1}{2} \sin z, f_{3}(z)=\frac{-\sqrt{3}}{2} \cos z-\frac{1}{2} \sin z$. It is easy to see the condition given in Theorem A is satisfied.

Very recently, Yang and Laine [3] present some studies on differential-difference analogues of Equation (1.2) showing that similar conclusions follow if one restricts the solutions to be of finite order. Yang and Laine [3] obtained:

Theorem B A nonlinear difference equation

$$
f^{3}(z)+q(z) f(z+1)=c \sin b z,
$$

where $q(z)$ is a nonconstant polynomial and $b, c \in \mathbb{C}$ are nonzero constants, does not admit entire solutions of finite order. If $q(z)=q$ is a nonzero constant, then Equation (1.3) possesses three distinct entire solutions of finite order, provided $b=3 \pi n$ and $q^{3}=(-1)^{n+1} \frac{27}{4} c^{2}$ for a nonzero integer $n$.

Theorem $\mathrm{C}$ Let $p, q$ be polynomials. Then a nonlinear difference equation

$$
f^{2}(z)+q(z) f(z+1)=p(z)
$$

has no transcendental entire solutions of finite order.

In this article, by the same method of [3], we replace $f(z+1)$ by $\Delta f(z)$ in Theorem B. We obtain:

Theorem 1 A nonlinear difference equation

$$
f^{3}(z)+q(z) \Delta f(z)=c \sin b z
$$

where $q(z)$ is a nonconstant polynomial and $b, c \in \mathbb{C}$ are nonzero constants, does not admit entire solutions of finite order. If $q(z)=q$ is a nonzero constant, then equation (1.3) possesses three distinct entire solutions of finite order, provided $b=3 \pi n$ ( $n$ must be odd integer) and $q^{3}=\frac{27}{32} c^{2}$ for a nonzero integer $n$. Without loss of generality, we may assume $\Delta f(z)=f(z+1)-f(z)$ in (1.4).

Example 1. In the special case of

$$
f^{3}(z)+\frac{3}{2}[f(z+1)-f(z)]=2 \sin 3 \pi z,
$$

a finite order entire solution is

$$
f_{1}(z)=-2 \sin \pi z=-\frac{1}{i}\left(e^{i \pi z}-e^{-i \pi z}\right) .
$$

The other two immediately follow from the conditions above:

$$
\begin{aligned}
& f_{2}(z)=\frac{1}{-i}\left(\varepsilon e^{i \pi z}-\varepsilon^{2} e^{-i \pi z}\right)=\sin \pi z-\sqrt{3} \cos \pi z \\
& f_{3}(z)=-\frac{1}{i}\left(\varepsilon^{2} e^{i \pi z}-\varepsilon e^{-i \pi z}\right)=\sin \pi z+\sqrt{3} \cos \pi z
\end{aligned}
$$

where $\varepsilon:=-\frac{1}{2}+\frac{\sqrt{3}}{2} i$ is a cubic of unity.

Theorem 2. A nonlinear difference equation

$$
f^{3}(z)+q(z) \Delta^{2} f(z)=c \sin b z,
$$


where $q(z)$ is a nonconstant polynomial and $b, c \in \mathbb{C}$ are nonzero constants, does not admit entire solutions of finite order. If $q(z)=q$ is a nonzero constant, then equation (1.5) possesses three distinct entire solutions of finite order, provided $b=3 \pi n(n$ must be odd integer) and $q^{3}=-\frac{27}{256} c^{2}$ for a nonzero integer $n$. Without loss of generality, we may assume $\Delta f(z)=f(z+1)-f(z)$ in (1.5).

We also replace $f(z+c)$ by $\Delta f(z)$ in the above Theorem $\mathrm{C}$. We obtain:

Theorem 3 Let $p, q$ be polynomials and let $m, n$ be positive integers satisfying $m \geq 3$. Then a nonlinear difference equation

$$
f^{m}(z)+q(z) \Delta^{n} f(z)=p(z)
$$

has no transcendental entire solutions of finite order. With out loss of generality, we may assume $\Delta f(z)=f(z+1)-f(z)$ in (1.6).

In 1959, Hayman [5] proposed:

Conjecture A If $f$ is a transcendental meromorphic function, then $f^{n} f$ assumes every finite non-zero complex number infinitely often for any positive integer $n$.

Hayman [5,6] himself confirmed it for $n \geq 3$ and for $n \geq 2$ in the case of entire $f$. Further, it was proved by Mues [7] when $n \geq 2$; Clunie [8] when $n \geq 1$ and $f$ is entire; Bergweiler and Eremenko [9] verified the case when $n=1$ and $f$ is of finite order, and finally by Chen and Fang [10] for the case $n=1$. For an analogue result in difference, in 2007, Laine and Yang [11] proved:

Theorem $\mathbf{D}$ Let $f$ be a transcendental entire function of finite order and $c$ be nonzero complex constant. Then for $n \geq 2, f(z)^{n} f(z+c)$ assumes every non-zero value $a$ $\in \mathbb{C}$ infinitely often.

Recently, Liu and Yang [12] improved Theorem D and obtained the next result.

Theorem $\mathbf{E}$ Let $f$ be a transcendental entire function of finite order, and $c$ be a nonzero complex constant. Then, for $n \geq 2 ; f(z)^{n} f(z+c)-p(z)$ has infinitely many zeros, where $p(z)$ is a non-zero polynomial.

Very recently, Qi et al. [13] obtained the following uniqueness theorem about the above results.

Theorem $\mathbf{F}$ [13] Let $f$ and $g$ be transcendental entire functions of finite order, and $c$ be a non-zero complex constant; let $n \geq 6$ be an integer. If $f^{n} f(z+c), g^{n} g(z+c)$ share $z \mathrm{CM}$, then $f=t_{1} g$ for a constant $t_{1}$ that satisfies $t_{1}^{n+1}=1$.

In the present paper, we get analogue results in difference, along with the following.

Theorem 4 Let $f$ be a transcendental meromorphic function of finite order $\rho$, and $\alpha$ $(z)$ be a small function with respect to $f(z)$. Suppose that $c$ is a nonzero complex constant and $\lambda, \mu$ are constants, $n, m$ are positive integers.

If $\lambda \neq 0$ and $n \geq 3 m+2$, then $f(z)^{n}\left(\mu f^{m}(z+c)+\lambda\right)-\alpha(z)$ has infinitely many zeros.

If $\lambda=0$ and $n+m \geq 3$, then $f(z)^{n}\left(\mu f^{m}(z+c)\right)-\alpha(z)$ has infinitely many zeros.

Theorem 5 Let $f$ and $g$ be transcendental entire functions of finite order, and $c$ be a non-zero complex constant. Suppose that and $\lambda, \mu$ are constants, $n, m$ are distinct positive integers.

If $\lambda \neq 0$ and $n \geq 4 m+5$ and $f(z)^{n}\left(\mu f(z+c)^{m}+\lambda\right), g(z)^{n}\left(\mu f(z+c)^{m}+\lambda\right)$ share $\alpha(z)$ $\mathrm{CM}$, then

1. $f(z) \equiv \operatorname{tg}(z)$ (where $t$ is a constant and $t^{n}=1$ ); or 
2. $f^{n}(z)\left(\mu f^{m}(z+c)+\lambda\right) g^{n}(z)\left(\mu g^{m}(z+c)+\lambda\right) \equiv \alpha^{2}(z)$

If $\lambda=0$ and $n+m \geq 9$ and $f(z)^{n}\left(\mu f(z+c)^{m}\right), g(z)^{n}\left(\mu f(z+c)^{m}\right)$ share $\alpha(z)$ CM, then

1. $f \equiv h_{1} g\left(h_{1}\right.$ is a constant and $\left.h_{1}^{n+m}=1\right)$; or

2. $f g=h_{2}\left(h_{2}\right.$ is a constant $)$.

Remark 2. Some ideas of this paper are based on $[3,13,14]$.

\section{Some Lemmas}

In order to prove our theorem, we need the following Lemmas:

As far as Clunie type lemmas are concerned, same conclusions hold as long as the proximity functions of the coefficients $\alpha(z)$ satisfy $m(r, \alpha)=S(r, f)$. The next lemma is a rather general variant of difference counterpart of the Clunie Lemma, see [15], for the corresponding results on differential polynomials, see [16].

Lemma 2.1 Let $f$ be a transcendental meromorphic solution of finite order $\rho$ of a difference equation of the form

$$
H(z, f) P(z, f)=Q(z, f),
$$

where $H(z, f), P(z, f), Q(z, f)$ are difference polynomials in $f$ such that the total degree of $H(z, f)$ in $f$ and its shifts is $n$, and the corresponding total degree of $Q(z, f)$ is $\leq n$. If $H(z, f)$ contains just one term of maximal total degree, then for any $\varepsilon>0$,

$$
m(r, P(z, f))=O\left(r^{\rho-1+\varepsilon}\right)+S(r, f),
$$

possibly outside of an exceptional set of finite logarithmic measure.

Lemma $2.2[17,18]$ Let $f$ be a transcendental meromorphic function of finite order $\rho$. Then for any given complex numbers $c_{1}, c_{2}$, and for each $\varepsilon>0$,

$$
m\left(r, \frac{f\left(z+c_{1}\right)}{f\left(z+c_{2}\right)}\right)=O\left(r^{\rho-1+\varepsilon}\right) .
$$

Lemma 2.3 [19] Suppose $c$ is a nonzero constant and $\alpha$ is a nonconstant meromorphic function. Then the differential equation $f^{2}+\left(c f^{(n)}\right)^{2}=\alpha$ has no transcendental meromorphic solutions satisfying $T(r, \alpha)=S(r, f)$.

Lemma 2.4 [17] Let $f$ be a meromorphic function of finite order $\rho$ and $c$ is a nonzero complex constant. Then, for each $\varepsilon>0$, We have

$$
T(r, f(z+c))=T(r, f)+O\left(r^{\rho-1+\varepsilon}\right)+O(\log r) .
$$

It is evident that $S(r, f(z+c))=S(r, f)$ from Lemma 2.4 .

Lemma 2.5 [17] Let $f$ be a meromorphic function with finite exponent of convergence of poles $\lambda\left(\frac{1}{f}\right)$ and $c$ is a non-zero complex constant. Then, for each $\varepsilon>0$, We have

$$
N(r, f(z+c))=N(r, f)+O\left(r^{\rho-1+\varepsilon}\right)+O(\log r) .
$$

Lemma 2.6 Let $f(z), n, m, \mu, \lambda$ and $c$ be as in Theorem 5. Denote $F(z)=f^{n}(z)\left(\mu f^{m}\right.$ $(z)+c)$. Then

$$
T(r, F)=(n+k) T(r, f)+S(r, f),
$$


where $k$ is a real constant and $k \in[-2 m, m]$.

Proof. It is easy to see $F(z)$ is not a constant. Otherwise, we may set $d=f^{n}(z)\left(\mu f^{m}\right.$ $(z+c)+\lambda)\left(d\right.$ is a constant). So $n T(r, f)=T\left(r, \mu f^{m}(z+c)+\lambda\right)+O(1)=m T$ $(r, f)+S(r, f)$. It is contradict with $m, n$ are distinct.

Case 1. $\lambda \neq 0$, Using Lemma 2.4, we have

$$
\begin{aligned}
T(r, F(z)) & =T\left(r, f^{n}(z)\left(\mu f^{m}(z)+c\right)\right) \leq n T(r, f(z))+m T(r, f(z+c)) \\
& \leq(n+m) T(r, f(z))+O\left(r^{\rho-1}\right)+S(r, f(z)) .
\end{aligned}
$$

On the other hand, using Lemma 2.2, we have

$$
\begin{aligned}
(n+m) T(r, f(z)) & =T\left(r, f^{n+m}(z)\right)+S(r, f(z)) \leq T\left(r, \frac{f^{n+m}(z)}{F(z)}\right)+T(r, F(z))+S(r, f(z)) \\
& \leq T\left(r, \frac{f^{n}(z)\left(\mu f^{m}(z+c)+\lambda\right)}{f^{n+m}(z)}\right)+T(r, F(z))+S(r, f(z)) \\
& \leq T\left(r, \frac{f^{m}(z+c)}{f^{m}(z)}\right)+m T(r, f)+T(r, F(z))+S(r, f(z)) \\
& \leq m m(r, f(z+c))+2 m T(r, f)+T(r, F(z))+S(r, f(z)) \\
& \leq m m\left(r, \frac{f(z+c)}{f(z)}\right)+3 m T(r, f)+T(r, F(z))+S(r, f(z))
\end{aligned}
$$

that is

$$
(n-2 m) T(r, f)+O\left(r^{\rho-1}\right)+S(r, f(z)) \leq T(r, F) \leq(n+m) T(r, f)+O\left(r^{\rho-1}\right)+S(r, f(z)) .
$$

So

$$
T(r, F)=(n+k) T(r, f)+S(r, f), \quad k \in[-2 m, m]
$$

Case 2. $\lambda=0$ By the same method of case 1 and Lemma 2.4, we obtain

$$
T(r, F)=(n+m) T(r, f)+S(r, f)
$$

The proof of Theorem 5 is complete.

Lemma 2.7 [20] Let $F$ and $G$ be two nonconstant meromorphic functions. If $F$ and $G$ share $1 \mathrm{CM}$, then one of the following three cases holds:

$$
\begin{aligned}
& \text { (1) } \max \{T(r, F), T(r, G)\} \leq N_{2}\left(r, \frac{1}{F}\right)+N_{2}\left(r, \frac{1}{G}\right)+N_{2}(r, F)+N_{2}(r, G)+S(r, F)+S(r, G) \text {; } \\
& \text { (2) } F=G ;(3) F G=1 .
\end{aligned}
$$

where $N_{2}\left(r, \frac{1}{F}\right)$ denotes the counting function of zeros of $F$ such that simple zeros are counted once and multiple zero twice.

\section{Proof of Theorem 1}

Let $f$ be an entire solution of Equation (1.4). Without loss of generality, we may assume that $f$ is transcendental entire.

Differentiating (1.4) results in

$$
3 f^{2}(z) f^{\prime}(z)+q^{\prime}(z) f(z+1)-q^{\prime}(z) f(z)+q(z) f^{\prime}(z+1)-q(z) f^{\prime}(z)=b c \cos b z .
$$

Combining (3.1) and (1.4), we get

$$
\begin{aligned}
& {\left[b f^{3}(z)+b q(z)(f(z+1)-f(z))\right]^{2}+\left[3 f^{2}(z) f^{\prime}(z)\right.} \\
& \left.\quad+q^{\prime}(z) f(z+1)-q^{\prime}(z) f(z)+q(z) f^{\prime}(z+1)-q(z) f^{\prime}(z)\right]^{2}=b^{2} c^{2} .
\end{aligned}
$$


This means that

$$
f^{4}(z)\left(b^{2} f^{2}(z)+9 f^{\prime 2}(z)\right)=T_{4}(z, f),
$$

where $T_{4}(z, f)$ is a differential-difference polynomial of $f$, of total degree at most 4 . If now $T_{4}(z, f)$ vanishes identically, then $f^{\prime}=i \frac{b}{3} f$ or $f^{\prime}=-i \frac{b}{3} f$, and therefore,

$$
f^{\prime \prime}+\left(\frac{b}{3}\right)^{2} f=0
$$

Otherwise, the Clunie lemma applied to a differential-difference equation, see Remark after Lemma 2.1, implies that

$$
T\left(r, b^{2} f^{2}+9\left(f^{\prime}\right)^{2}\right)=m\left(r, b^{2} f^{2}+9\left(f^{\prime}\right)^{2}\right)=S(r, f) .
$$

Therefore, $\alpha:=b^{2} f^{2}+9(f)^{2}$ is a small function of $f$, not vanishing identically. By Lemma 2.3, $\alpha$ must be a constant. Differentiating $b^{2} f^{2}+9(f)^{2}=\alpha$, we immediately conclude that (3.3) holds in this case as well. Solving (3.3) shows that $f$ must be of the form

$$
f(z)=c_{1} \mathrm{e}^{\frac{i b z}{3}}+c_{2} \mathrm{e}^{-\frac{i b z}{3}} .
$$

Substituting the preceding expression of $f$ into the original difference equation (1.4), expressing $\sin b z$ in the terms of exponential function, and denoting $w(z):=\mathrm{e}^{\frac{i b z}{3}}$, an elementary computation results in

$$
\left(c_{1}^{3}+\frac{i c}{2}\right) w^{6}+\left(3 c_{1}^{2} c_{2}+c_{1} q(z) e^{\frac{i b}{3}}-c_{1} q(z)\right) w^{4}+\left(3 c_{1} c_{2}^{2}+c_{2} q(z) e^{\frac{-i b}{3}}-c_{2} q(z)\right) w^{2}+c_{2}^{3}-\frac{i c}{2}=0,
$$

where

$$
a_{6}=c_{1}^{3}+\frac{1}{2} i c, a_{4}=3 c_{1}^{2} c_{2}+c_{1} q(z) e^{\frac{i b}{3}}-c_{1} q(z), a_{2}=3 c_{1} c_{2}^{2}+c_{2} q(z) \mathrm{e}^{\frac{-i b}{3}}-c_{2} q(z), a_{0}=c_{2}^{3}-\frac{1}{2} i c .
$$

Since $w(z)$ is transcendental, we must have $a_{0}=a_{2}=a_{4}=a_{6}=0$. Therefore, $c_{1} \neq 0$, $c_{2} \neq 0$, and the condition $a_{4}=0$ implies that $q(z)$ is a constant, say $q \neq 0$. Combing now the conditions $a_{4}=0$ and $a_{2}=0$ we conclude that $q\left(e^{\frac{i b}{3}}-e^{-\frac{i b}{3}}\right)=0$, and then $\mathrm{e}^{\frac{2 i b}{3}}=1=\mathrm{e}^{2 \pi i n}$, hence $b=3 \pi n$. The connection between $q$ and $c$ now follows from $3 c_{1} c_{2}+(-1)^{n} q-q=0$ and $\left(c_{1} c_{2}\right)^{3}=\frac{1}{4} c^{2}$. We obtain, $27 c_{1}^{3} c_{2}^{3}=q^{3}\left[1-(-1)^{n}\right]^{3}$, so $n$ must be an odd. Finally, $q^{3}=\frac{27}{32} c^{2}$. The proof of Theorem 1 is complete.

\section{Proof of Theorem 2}

Due to the same idea of Proof Theorem 1, we omit the proof.

\section{Proof of Theorem 3}

Suppose that $f$ is a transcendental entire solution of finite order $\rho$ to Equation (1.6). Without loss of generality, we may assume that $q(z)$ does not vanish identically. From (1.6), we readily conclude by Lemma 2.2 that

$$
\begin{aligned}
m m(r, f) & =m\left(r, p(z)-q(z) \Delta^{n} f(z)\right) \leq m\left(r, \Delta^{n} f(z)\right)+O(\log r) \\
& \leq m\left(r, \frac{f(z+n)}{f(z)}\right)+m\left(r, \frac{f(z+n-1)}{f(z)}\right)+\cdots+m\left(r, \frac{f(z+1)}{f(z)}\right)+2 m(r, f)+O(\log r), \\
& =2 m(r, f)+O\left(r^{\rho-1+\varepsilon}\right)+O(\log r),
\end{aligned}
$$


and then

$$
(m-2) T(r, f)=(m-2) m(r, f) \leq O\left(r^{\rho-1+\varepsilon}\right)+O(\log r) .
$$

By the $m \geq 3$, hence $\rho(f)<\rho$, a contradiction.

\section{Proof of Theorem 4}

Case 1. If $\lambda \neq 0$, then we denote $F(z)=f(z)^{n}\left(\mu f(z+c)^{m}+\lambda\right)$. From Lemma 2.6, we have (2.1) and $F(z)$ is not a constant. Since $f(z)$ is a transcendental entire function of finite order, we get $T(r, f(z+c))=T(r, f(z))+S(r, f)$ from Lemma 2.4. By the second main theorem, we have

$$
\begin{aligned}
(n+k) T(r, f(z)) & =T(r, F(z))+S(r, f) \\
& \leq \bar{N}(r, 1 / F(z))+\bar{N}(r, 1 /(F(z)-\alpha(z))+S(r, f) \\
& \leq \bar{N}(r, 1 / f(z))+\bar{N}\left(r, 1 /\left(f^{m}(z+c)+\lambda / \mu\right)\right)+\bar{N}(r, 1 /(F(z)-\alpha(z)) \\
& \leq(m+1) T(r, f(z))+\bar{N}(r, 1 /(F(z)-\alpha(z))+S(r, f) .
\end{aligned}
$$

Thus

$$
(n+k-m-1) T(r, f(z)) \leq \bar{N}(r, 1 /(F(z)-\alpha(z))+S(r, f)
$$

The assertion follows by $n \geq 3 m+2$.

Case 2. If $\lambda=0$, then we denote $F(z)=f(z)^{n}\left(\mu f(z+c)^{m}\right)$. From Lemma 2.6, we have (2.2) and $F(z)$ is not a constant,

$$
\begin{aligned}
(n+m) T(r, f(z)) & =T(r, F(z))+S(r, f) \\
& \leq \bar{N}(r, 1 / F(z))+\bar{N}(r, 1 /(F(z)-\alpha(z))+S(r, f) \\
& \leq \bar{N}(r, 1 / f(z))+\bar{N}(r, 1 /(f(z+c)))+\bar{N}(r, 1 /(F(z)-\alpha(z))+S(r, f) \\
& \leq 2 T(r, f)+\bar{N}(r, 1 /(F(z)-\alpha(z))+S(r, f) .
\end{aligned}
$$

Thus

$$
(n+m-2) T(r, f(z)) \leq \bar{N}(r, 1 /(F(z)-\alpha(z))+S(r, f) .
$$

The assertion follows by $n+m \geq 3$. The Proof of Theorem 5 is complete.

\section{Proof of Theorem 5}

Case $1 \lambda \neq 0$. Denote

$$
F(z)=\frac{f(z)^{n}\left(\mu f(z+c)^{m}+\lambda\right)}{\alpha(z)}, \quad G(z)=\frac{g(z)^{n}\left(\mu g(z+c)^{m}+\lambda\right)}{\alpha(z)} .
$$

Then $F(z)$ and $G(z)$ share 1 CM except the zeros or poles of $\alpha(z)$, and

$$
\begin{aligned}
& T(r, F)=(n+k) T(r, f)+S(r, f), \quad k \in[-2 m, m] \\
& T(r, G)=(n+k) T(r, g)+S(r, g), \quad k \in[-2 m, m]
\end{aligned}
$$

from Lemma 2.6. By the definition of $F$, we get $N_{2}(r, F(z))=S(r, f)$ and

$$
\begin{aligned}
N_{2}(r, 1 / F(z)) & \leq 2 \bar{N}(r, 1 / f(z))+N\left(r, 1 /\left(f^{m}(z+c)+\lambda / \mu\right)\right) \\
& \leq 2 T(r, f(z))+m T(r, f(z+c))+S(r, f(z)) \\
& \leq(m+2) T(r, f)+S(r, f) .
\end{aligned}
$$


Then

$$
N_{2}(r, F)+N_{2}(r, 1 / F) \leq(m+2) T(r, f)+S(r, f) .
$$

Similarly,

$$
N_{2}(r, G)+N_{2}(r, 1 / G) \leq(m+2) T(r, g)+S(r, g) .
$$

Suppose that (2.3) hold. Substituting (7.4) and (7.5) into (2.3), we obtain

$$
\max \{T(r, F), T(r, G)\} \leq(m+2)(T(r, f)+T(r, g))+S(r, f)+s(r, g) .
$$

Then

$$
T(r, f)+T(r, g) \leq(2 m+4)\{T(r, f(z))+T(r, g(z))\}+S(r, f)+S(r, g) .
$$

Substituting (7.2) and (7.3) into the last inequality, yields

$$
(n+k-2 m-4)\{T(r, F)+T(r, G)\} \leq S(r, f)+S(r, g),
$$

contradicting with $n \geq 4 m+5$. Hence $F(z) \equiv G(z)$ or $F(z) G(z) \equiv \alpha(z)^{2}$ by Lemma 2.7, We discuss the following two subcases.

Subcase 1.1. Suppose that $F(z) \equiv G(z)$. That $f(z)^{n}\left(\mu f(z+c)^{m}+\lambda\right) \equiv g(z)^{n}(\mu g(z+c)$ $m+\lambda)$. Let $h(z)=f(z) / g(z)$. If $h(z)^{n} h^{m}(z+c) \otimes 1$, we have

$$
g^{m}(z+c)=\frac{\lambda}{\mu} \frac{1-h(z)^{n}}{h(z)^{n} h^{m}(z+c)-1} .
$$

Then $h$ is a transcendental meromorphic function with finite order since $g$ is transcendental. By Lemma 2.4, we have

$$
T(r, h(z+c))=T(r, h(z))+S(r, h) .
$$

By the condition $n \geq 4 m+5$, it is easily to show that $h(z)^{n} h^{m}(z+c)$ is not a constant from (7.7).

Suppose that there exists a point $z_{0}$ such that $h\left(z_{0}\right)^{n} h^{m}\left(z_{0}+c\right)=1$. Then $h\left(z_{0}\right)^{n}=1$ from (7.6) since $g(z)$ is entire function. Hence $h^{m}\left(z_{0}+c\right)=1$ and

$$
\bar{N}\left(r, 1 /\left(h(z)^{n} h^{m}(z+c)-1\right)\right) \leq \bar{N}\left(r, 1 / h^{m}(z+c)-1\right) \leq m T(r, h(z))+S(r, h)
$$

Denote $H:=h(z)^{n} h^{m}(z+c)$, from the above inequality and (7.7), we apply the second main theorem to $H$, resulting in

$$
\begin{aligned}
T(r, H) & \leq \bar{N}(r, H)+\bar{N}\left(r, \frac{1}{H}\right)+\bar{N}\left(r, \frac{1}{H-1}\right)+S(r, h) \\
& \leq \bar{N}\left(r, 1 /\left(h(z)^{n} h^{m}(z+c)\right)\right)+N\left(r,\left(h(z)^{n} h^{m}(z+c)\right)+m T(r, h(z))+S(r, h)\right. \\
& \leq \bar{N}(r, h)+\bar{N}(r, h(z+c))+\bar{N}(r, 1 / h)+\bar{N}(r, 1 / h(z+c))+m T(r, h(z))+S(r, h) \\
& \leq(m+4) T(r, h)+S(r, h) .
\end{aligned}
$$

Noting this, we have

$$
\begin{aligned}
n T(r, h) & =T\left(r, \frac{H}{h^{m}(z+c)}\right) \leq T(r, H)+T\left(r, h^{m}(z+c)\right)+O(1) \\
& \leq(2 m+4) T(r, h)+O\left(r^{\rho-1+\varepsilon}\right)+S(r, h),
\end{aligned}
$$

and then

$$
(n-2 m-4) T(r, h) \leq O\left(r^{\rho-1+\varepsilon}\right)+S(r, h),
$$


which is a contradiction since $n \geq 4 m+5$. Therefore, $h(z)^{n} h(z+c)^{m} \equiv 1$. Thus $h^{n}(z) \equiv 1$. Hence $f(z) \equiv \operatorname{tg}(z)$, where $t$ is a constant and $t^{n}=1$.

Subcase 1.2. Suppose that $F(z) G(z) \equiv \alpha(z)^{2}$. That is

$$
f^{n}(z)\left(\mu f^{m}(z+c)+\lambda\right) g^{n}(z)\left(\mu g^{m}(z+c)+\lambda\right) \equiv \alpha^{2}(z) .
$$

Case 2. $\lambda=0$. Denote

$$
F(z)=\frac{f(z)^{n}\left(\mu f(z+c)^{m}\right)}{\alpha(z)}, G(z)=\frac{g(z)^{n}\left(\mu g(z+c)^{m}\right)}{\alpha(z)} .
$$

Then $F(z)$ and $G(z)$ share 1 CM except the zeros or poles of $\alpha(z)$, and

$$
\begin{aligned}
& T(r, F)=(n+m) T(r, f)+S(r, f), \\
& T(r, G)=(n+m) T(r, g)+S(r, g),
\end{aligned}
$$

from Lemma 2.6. By the definition of $F$, we get $N_{2}(r, F(z))=S(r, f)$ and

$$
\begin{aligned}
N_{2}(r, 1 / F(z)) & \leq 2 \bar{N}(r, 1 / f(z))+2 \bar{N}(r, 1 /(f(z+c)) \\
& \leq 2 T(r, f(z))+2 T(r, f(z+c))+S(r, f(z)) \\
& \leq 4 T(r, f)+S(r, f) .
\end{aligned}
$$

Then

$$
N_{2}(r, F)+N_{2}(r, 1 / F) \leq 4 T(r, f)+O\left(r^{\rho-1+\varepsilon}\right)+S(r, f) .
$$

Similarly,

$$
N_{2}(r, G)+N_{2}(r, 1 / G) \leq 4 T(r, g)+O\left(r^{\rho-1+\varepsilon}\right)+S(r, g) .
$$

Suppose that (2.3) hold. Substituting (7.11) and (7.12) into (2.3), we obtain

$$
\max \{T(r, F), T(r, G)\} \leq 4(T(r, F)+T(r, G))+S(r, f)+s(r, g) .
$$

Then

$$
T(r, F)+T(r, G) \leq 8\{T(r, f(z))+T(r, g(z))\}+S(r, f)+S(r, g) .
$$

Substituting (7.9) and (7.10) into the last inequality, yields

$$
(n+m-8)\{T(r, F)+T(r, G)\} \leq S(r, f)+S(r, g),
$$

contradicting with $n+m \geq 9$. Hence $F(z) \equiv G(z)$ or $F(z) G(z) \equiv \alpha(z)^{2}$ by Lemma 2.7. We discuss the following two subcases.

subcase 2.1. Suppose that $F(z) \equiv G(z)$. That $f(z)^{n}\left(\mu f(z+c)^{m}\right) \equiv g(z)^{n}\left(\mu g(z+c)^{m}\right)$. Let $h_{1}(z)=f(z) / g(z)$. If $h_{1}(z)$ is not a constant, we have

$$
h_{1}(z)^{n} h_{1}(z+c)^{m} \equiv 1 \text {. }
$$

Then $h_{1}$ is a meromorphic function with finite order since $g$ and $f$ are of finite order. By Lemma 2.4 and (7.13), we have

$$
m T\left(r, h_{1}(z+c)\right)=n T\left(r, h_{1}(z)\right)+S\left(r, h_{1}\right),
$$

and then

$$
m=n,
$$


a contradiction. So $h_{1}(z)$ must be a constant, then $f \equiv h_{1} g\left(h_{1}\right.$ is a constant and $\left.h^{n+m}=1\right)$. subcase 2.2. Suppose that $F(z) G(z) \equiv \alpha(z)^{2}$. That is

$$
f^{n}(z)\left(\mu f^{m}(z+c)\right) g^{n}(z)\left(\mu g^{m}(z+c)\right) \equiv \alpha^{2}(z) .
$$

Let $f(z) g(z)=h_{2}(z)$, By a reasoning similar to that mentioned at the end of the proof of Case2.1, we know that $h_{2}(z)$ must be a constant, then $f g=h_{2}\left(h_{2}\right.$ is a constant). The proof of Theorem 6 is complete.

\section{Acknowledgements}

The authors are grateful to the referees for their valuable suggestions and comments. The authors would like to express their hearty thanks to Professor Hongxun Yi for his valuable advice and helpful information. Supported by project 10XKJ01 from Leading Academic Discipline Project of Shanghai Dianji University and also supported by the NSFC (No.10771121, No.10871130), the NSF of Shandong (No. Z2008A01) and the RFDP (No.20060422049), and The National Natural Science Youth Fund Project (51008190).

\section{Author details}

${ }^{1}$ Department of Mathematics and Physics, Shanghai Dianji University, Shanghai 200240, PR China ${ }^{2}$ Department of Mathematics, Shandong University, Jinan 250100, PR China

\section{Authors' contributions}

JQ drafted the manuscript and have made outstanding contributions to this paper. JD and TZ participated in the sequence alignment.

\section{Competing interests}

The authors declare that they have no competing interests.

Received: 25 February 2011 Accepted: 6 September 2011 Published: 6 September 2011

\section{References}

1. Yang, CC, Yi, HX: Uniqueness Theory of Meromorphic Functions. Kluwer Academic Publishers, Norwell (2003)

2. Yang, L: Value distribution theory. Springer, Berlin (1993)

3. Yang, CC, Laine, I: On analogies between nolinear difference and differential equations. Proc Jpn Acad Ser A. 86, 10-14 (2010). doi:10.3792/pjaa.86.10

4. Li, P, Yang, CC: On the nonexistence of entire solutions of certain type of linear differential equations. J Math Anal Appl. 320(2), 827-835 (2006). doi:10.1016/j.jmaa.2005.07.066

5. Hayman, WK: Picard value of meromorphic functions and their derivatives. Ann Math. 70, 9-42 (1959). doi:10.2307/ 1969890

6. Hayman, WK: Research problem in function theory. Athlone Press, University of London (1967)

7. Mues, E: über ein problem von Hayman. Math Z. 164, 239-259 (1979). doi:10.1007/BF01182271

8. Clunie, J: On a result of Hayman. J Lond Math Soc. 42, 389-392 (1967). doi:10.1112/jlms/s1-42.1.389

9. Bergweiler, W, Eremenko, A: Complex dynamics and value distribution. Intenational Conference of Complex Analysis, Nanjing. (1994)

10. Chen, HH, Fang, ML: On the value distribution of $f^{n} f^{\prime}$. Sci China Ser A. 38, 789-798 (1995)

11. Laine, I, Yang, CC: Value distribution of difference polynomials. Proc Jpn Acad Ser A. 83, 148-151 (2007). doi:10.3792/ pjaa.83.148

12. Liu, K, Yang, LZ: Value distribution of the difference operator. Arch Math. 92, 270-278 (2009). doi:10.1007/s00013-0092895-X

13. Qi, XG, Yang, LZ, Liu, K: Uniqueness and periodicity of meromorphic functions concerning the difference operator. Comput Math Appl. 60, 1739-1746 (2010). doi:10.1016/j.camwa.2010.07.004

14. Zhang, JL: Value distribution and shared sets of difference of meromorphic functions. J Math Anal Appl. 367, 401-408 (2010). doi:10.1016/j.jmaa.2010.01.038

15. Laine, I, Yang, CC: Clunie theorems for difference and q-difference polynomials. J Lond Math Soc. 76(2), 556-566 (2007)

16. Yang, CC, Ye, Z: Estimates of the proximate function of differential polynomials. Proc Jpn Acad Ser A Math Sci. 83(4), 50-55 (2007). doi:10.3792/pjaa.83.50

17. Chiang, YM, Feng, SJ: On the Nevanlinna characteristic of $f(z+\eta)$ and difference equations in the complex plane. Ramanujan J.16(1), 105-129

18. Halburd, RG, Korhonen, RJ: Difference analogue of the lemma on the logarithmic derivative with application to difference equations. J Math Anal Appl. 314(2), 477-487 (2006). doi:10.1016/j.jmaa.2005.04.010

19. Heittokangas, J, Korhonen, R, Laine, I: On meromorphic solutions of certain non-linear differntial equations. Bull Austral Math Soc. 66(2), 331-343 (2002). doi:10.1017/S000497270004017X

20. Yang, CC, Hua, XH: Uniqueness and value-sharing of meromorphic functions. Ann Acad Sci Fenn Math. 22, 395-406 (1997)

doi:10.1186/1029-242X-2011-50

Cite this article as: Qi et al:: Some results about a special nonlinear difference equation and uniqueness of difference polynomial. Journal of Inequalities and Applications 2011 2011:50. 